\title{
Less May Be Best: High Flow Rates Are Not Always the Answer
}

\author{
Jie $\mathrm{Li}^{1}, \mathrm{~J}$ Scott${ }^{1}$, and Jan Hau Lee ${ }^{2}$ \\ ${ }^{1}$ Rush University \\ ${ }^{2}$ KK Women's and Children's Hospital
}

August 14, 2020

\section{References}

1. Luo J, Duke T, Chisti MJ, Kepreotes E, Kalinowski V, Li J. Efficacy of high-flow nasal cannula vs standard oxygen therapy or nasal continuous positive airway pressure in children with respiratory distress: A meta-analysis. J Pediatr. 2019;215:199-208

2. Li J, Jing GQ, Scott JB. Year in Review 2019: High-flow nasal cannula (HFNC) oxygen therapy for adult patients. Respir Care, 2020, 65(4): 545-557.

3. Lavizzari A, Veneroni C, Colnaghi M, et al. Respiratory mechanics during NCPAP and HHHFNC at equal distending pressures. Arch Dis Child Fetal Neonatal Ed. 2014;99(4):F315-F320.

4. Simon M, Braune S, Frings D, Wiontzek AK, Klose H, Kluge S. High-flow nasal cannula oxygen versus non-invasive ventilation in patients with acute hypoxaemic respiratory failure undergoing flexible bronchoscopy-a prospective randomised trial. Crit Care 2014;18(6):712. doi: 10.1186/s13054-014-07129.

5. Saksitthichok B, Petnak T, So-Ngern A, Boonsarngsuk V. A prospective randomized comparative study of high-flow nasal cannula oxygen and non-invasive ventilation in hypoxemic patients undergoing diagnostic flexible bronchoscopy. J Thorac Dis 2019;11(5):1929-1939.

6. Douglas N, Ng I, Nazeem F, et al. A randomised controlled trial comparing high-flow nasal oxygen with standard management for conscious sedation during bronchoscopy. Anaesthesia 2018;73(2):169-176

7. Lin Y, Zhang X, Li L, et al. High-flow nasal cannula oxygen therapy and hypoxia during gastroscopy with propofol sedation: A randomized multicenter clinical trial. Gastrointest Endosc 2019;90(4):591601.

8. Teng WN, Ting CK, Wang YT, et al. High-flow nasal cannula and mandibular advancement bite block decrease hypoxic events during sedative esophagogastroduodenoscopy: A randomized clinical trial. Biomed Res Int 2019:4206795.

9. Riccio CA, Sarmiento S, Minhajuddin A, Nasir D, Fox AA. High-flow versus standard nasal cannula in morbidly obese patients during colonoscopy: A prospective, randomized clinical trial. J Clin Anesth 2019;54:19-24.

10. Nielsen KR, Ellington LE, Gray AJ, Stanberry LI, Smith LS, DiBlasi RM. Effect of high-flow nasal cannula on expiratory pressure and ventilation in infant, pediatric, and adult models. Respir Care 2018;63:147-57.

11. Coté CJ, Wilson S; American academy of pediatrics; American academy of pediatric dentistry. Guidelines for monitoring and management of pediatric patients before, during, and after sedation for diagnostic and therapeutic procedures. Pediatrics. 2019;143(6):e20191000.

12. Klotz D, Seifert V, Baumgartner J, Teufel U, Fuchs H. High-flow nasal cannula vs standard respiratory care in pediatric procedural sedation: A randomized controlled pilot trial. Pediatr Pulmonol. 2020;1-7.

13. Scott JB, Kaur R. Monitoring breathing frequency, pattern, and effort. Respir Care. 2020;65(6):793806.

14. Neuman Y, Koslow M, Matveychuk A, Bar-Sef A, Guber A, Shitrit D. Increased hypoxemia in patients 
with COPD and pulmonary hypertension undergoing bronchoscopy with biopsy. Int J Chron Obstruct Pulmon Dis. 2015;10:2627-2632. 